\title{
PRETERM PREMATURE RUPTURE OF THE MEMBRANES AND GENITAL MYCOPLASMAS
}

\author{
Marian Kacerovský ${ }^{1}$, Michal Pavlovský2, Jindřich Tošner ${ }^{1}$
}

Charles University in Prague, Faculty of Medicine and University Hospital Hradec Králové, Czech Republic: Department of Obstetrics and Gynecology1; Hospital Most, Czech Republic: Department of Pathology ${ }^{2}$

\begin{abstract}
Summary: Objective: The purpose of this study was to evaluate the prevalence of cervical colonization by genital mycoplasmas in patients with preterm premature rupture of the membranes (PPROM). Method: We studied 225 women between 24 and 36 weeks of gestation with PPROM. Cervical swabs were obtained for genital mycoplasmas and standard vaginal smears of bacterial culture were performed at the time of patients' admission. In the control group were 225 women with a normal pregnancy. Results: Ureaplasma urealyticum was detected in $68 \%(152 / 225)$ and Mycoplasma hominis was detected in $28 \%(63 / 225)$ of the patients with PPROM between 24 and 36 weeks of gestation and. In the control group Ureaplasma urealyticum was found in $17 \%$ (38/225) and Mycoplasma hominis in $15 \%$ (35/225) pregnant women. Conclusion: Our results provide evidence of an association between cervical colonization with genital mycoplasmas and preterm premature rupture of the membranes.
\end{abstract}

Key words: Cervical colonization; Genital mycoplasmas; Preterm premature rupture of the membranes

\section{Introduction}

Preterm delivery is one of the main causes of perinatal mortality and morbidity worldwide. It is an escalating problem in Czech Republic and has increased to $6 \%$. Premature rupture of the membranes is defined as fetal membranes rupture with leakage of amniotic fluid that precedes the onset of uterine contraction by at least 2 hours. It complicates 4-7 \% of all births and is directly associated with a short length of gestation and an increased perinatal morbidity and mortality. There are predisposing conditions connected with the occurrence of premature rupture of the membranes such as local infections, cervical incompetence and low socio-economic condition $(5,13)$. Even so, the aetiology still remains unknown in the majority of cases. Increased biosynthesis of prostaglandins by intrauterine tissue is widely accepted as a key event in the initiation of parturition (8). Preterm labour after spontaneous rupture of the membranes is associated with a significant increase in amniotic fluid concentration of prostaglandins. A main reason of this elevation is probably infection. The purpose of this study was to evaluate the prevalence of cervical colonization by genital mycoplasmas (Ureaplasma urealyticum and/or Mycoplasma hominis) in patients with preterm premature rupture of the membranes (PPROM). The cervical detection rates of genital mycoplasmas were compared with a normal population of pregnant women in the Czech Republic.

\section{Patients and method}

\section{Study population}

The study population involved 225 pregnant women with PPROM (gestation age between 24 and 36 weeks) who were admitted to the department of Obstetrics and Gynaecology in Most from January 2004 to February 2007. There were no differences in the demographic data of the groups studied and all groups were similar with regard to parity and gravidity. Their age at the time of diagnosis ranged from 19 to 38 years (median age 26). Women with multiple gestation or major fetal anomaly were excluded from the study. Written consent was obtained from each patient. Rupture of membranes was diagnosed by an examination with a sterile speculum and a combination of vaginal pooling of amniotic fluid and the nitrazine test or a positive result for insulin-like growth factor binding protein - 1 (PROM test) in the vaginal fluid. Cervical swabs were obtained for detecting genital mycoplasmas and standard vaginal smears of bacterial culture were performed at the time of their admission. Sample of maternal blood was obtained for investigation of maternal CRP level. The control group comprised 225 otherwise healthy women in the mid trimester of pregnancy (gestation age between 24-28 weeks) from whom we obtained cervical swabs for genital mycoplasmas during routine prenatal examination. All these women delivered between 38 th and 41 st week of gestation without ad- 
verse neonatal outcomes. Clinical management in the study group was performed according to standard protocols at our department. The antibiotics were given after collecting the cervical swab and the vaginal smear.

\section{Statistical methods}

Statistical analyses were performed using Fischer's exact tests. A probability value of $<0.05$ was considered significant.

\section{Culture procedures}

The Mycoplasma IST 2 kit (Biomérieux, France) was used to diagnose the mycoplasmal infections. It enables culture, identification, indicative enumeration and antibiotic susceptibility testing (with 9 antibiotics - doxycycline, josamycin, ofloxacin, erythromycin, tetracycline, ciprofloxacin, azitromycin, clarytromycin and pristinamycin) of Ureaplasma urealyticum (Uu.) and Mycoplasma hominis (Mh.). It combines a selective culture broth with a strip containing 22 testes. This kit allows pathogen identification within 48 hours and determines the amount of bacteria, thus making differentiation possible between colonization and infection (cell count above $10^{4}$ is the evidence of infection). The combination of three antibiotics and one antifungal agent provides selectivity, ensuring that any contamining flora present in the specimen does not affect the test. After the collecting the sample the cervical swab for $\mathrm{Uu}$. and $\mathrm{Mh}$. was inserted into the Biomérieux transport medium R1, provided with Mycoplasma IST 2 kit. The swab was stored in the transport medium at $4{ }^{\circ} \mathrm{C}$ until transported to the laboratory. All the swabs were processed in

Tab. 1: Colonization of the cervix with the genital mycoplasmas $(\mathrm{p}<0.0001)$.

\begin{tabular}{|l|c|c|}
\hline & $\begin{array}{c}\text { Group with } \\
\text { PPROM }\end{array}$ & $\begin{array}{c}\text { Control } \\
\text { group }\end{array}$ \\
\hline Uu. Positive & $152(68 \%)$ & $38(17 \%)$ \\
\hline Mh. Positive & $63(28 \%)$ & $35(15 \%)$ \\
\hline Uu. and/or Mh. negative & $10(4 \%)$ & $152(68 \%)$ \\
\hline
\end{tabular}

the laboratory within 5 hours of collection. The results were interpreted after 24 hours and 48 hours of incubation.

\section{Diagnosis of amnionitis chorioamnionitis, and funisitis}

Histologic chorioamnionitis was defined as the presence of acute inflammatory changes on examination of a membrane roll and chorionic plate of the placenta; funisitis was diagnosed in the presence of neutrophil infiltration into the umbilical vessel walls or Wharton's jelly. Amnionitis was defined as the presence of acute inflammatory changes in the membranes.

\section{Results}

\section{Colonization of the cervix with genital mycoplasmas}

In the normal pregnancy group, $32 \%(73 / 225)$ of the women in this group had a positive cervical culture for genital mycoplasmas. In contrast, the cervical colonisation ratio for genital mycoplasmas in women with PPROM was $96 \%(215 / 225)$ - Tab. 1, demonstrating a significant difference between groups with PPROM and control group ( $\mathrm{p}<$ 0.0001 , by Fisher's exact test).

\section{Maternal serum $C$ - reactive protein in cases with PPROM}

Maternal serum C - reactive protein (CRP) levels were divided into 3 group: negative $(0-5 \mathrm{mg} / 1)$, mild positive (6-19 $\mathrm{mg} / \mathrm{l})$ and positive (20 and more $\mathrm{mg} / \mathrm{l})$. A negative CRP was encountered in $56 \%(126 / 225)$, mild positive in $10 \%(22 / 225)$ and a positive result was found in $34 \%$ $(77 / 225)$

\section{Comparison of maternal serum $C$-reactive protein in cases of PPROM and histopathologic findings on the placenta and the membranes}

We compared maternal serum CRP in cases of PPROM and histopathological findings on the placenta and the

Tab. 2: Comparison of maternal serum CRP in cases of PPROM and histopathologic findings on the placenta and the membranes $(\mathrm{p}<0.0138)$.

\begin{tabular}{|l|c|c|c|c|}
\hline & $\begin{array}{c}\text { Without inflamma- } \\
\text { tory changes }\end{array}$ & Amnionitis & Chorioamnionitis & Funisitis \\
\hline Negative CRP & $56(44 \%)$ & $22(18 \%)$ & $19(15 \%)$ & $29(23 \%)$ \\
\hline Mild positive CRP & $7(32 \%)$ & $0(0 \%)$ & $11(50 \%)$ & $4(18 \%)$ \\
\hline Positive CRP & $24(31 \%)$ & $1(1 \%)$ & $24(31 \%)$ & $28(37 \%)$ \\
\hline
\end{tabular}

Tab. 3: The histopathologic findings on the placenta and the membranes in cases of PPROM with positive genital mycoplasmas $(\mathrm{p}<0.0047)$.

\begin{tabular}{|l|c|c|c|c|}
\hline & $\begin{array}{c}\text { Without inflamma- } \\
\text { tory changes }\end{array}$ & Amnionitis & Chorioamnionitis & Funisitis \\
\hline Positive genital mycoplasmas & $81(36 \%)$ & $19(8 \%)$ & $54(24 \%)$ & $61(27 \%)$ \\
\hline Negative genital mycoplasmas & $6(3 \%)$ & $4(2 \%)$ & $0(0 \%)$ & $0(0 \%)$ \\
\hline
\end{tabular}


membranes. Patients with a negative serum CRP did not have inflammatory changes on the placenta and the membranes in $44 \%(56 / 126)$; amnionitis was found in $18 \%$ $(22 / 126)$ chorioamnionitis was found in $15 \%(19 / 126)$, funisitis in $23 \%(29 / 126)$. In patients with a positive serum CRP; $31 \%$ (24/77) did not have any inflammatory changes; amnionitis was in $1 \%(1 / 77)$ chorioamnionitis was in $31 \%$ $(24 / 77)$ and funisitis in $37 \%(28 / 77)$ (Tab. 2). A statistical difference was found ( $\mathrm{p}<0.0138$, by Fischer's exact test).

\section{Histopathologic findings on the placenta and the membranes in cases of PPROM with positive genital mycoplasmas}

The prevalence of chorioamnionitis and funisitis was $25 \%$ $(54 / 215)$ and $28 \%(61 / 215)$ respectively in cases of PPROM with positive genital mycoplasmas (Tab. 3). A statistical difference was found $(p<0.0047$, by Fischer's exact test).

\section{Discussion}

Genital mycoplasmas compose the microorganisms that are the most frequently isolated from both placental membranes and amniotic fluid in women with histological and clinical chorioamnionitis (5). These organisms are commonly found within the uterus in association with spontaneous preterm labor and with PPROM (13). The intrauterine presence of these organisms have been associated with an increased production of a wide variety of cytokines, matrix metalloproteinases, and prostaglandins, all believed to be in the causal pathway and/or precursors for spontaneous labor and PPROM (8). Ureaplasma urealyticum (Uu.) has been detected in the lower genital tract of $40 \%$ to $80 \%$ sexually active women depending on geographical areas, and appears to be common in the vaginal flora of pregnant women (6). Witt et al described microbial invasion of the amniotic cavity with Uu. in $43.9 \%$ patients with preterm labor (14). Vice versa genital mycoplasmas are the organisms most frequently isolated from the amniotic fluid in patients with preterm and term gestations, and they have been implicated in the pathogenesis of clinical chorioamnionitis, puerperal endometritis, postoperative wounds infections, neonatal sepsis, meningitis and in the genesis of bronchopulmonaly dysplasia $(2,12)$. Mc Donald et al. described a colonization ratio with Uu. of $35 \%$ in midtrimester pregnancy and found that colonized women had a risk for preterm labor almost twice as high and for PPROM more than 3-fold that of women with negatives cultures (9). Goldenberg et al. found that in preterm deliveries (infants delivered between 23-32 weeks), samples taken from the umbilical cord had a positive culture for Ureaplasma urealyticum and Mycoplasma hominis. in $23.4 \%$. Positive cultures were more commonly present in non white (predominantly African - American) women and in women less than 20 years of age. Infants with cord blood positive for genital mycoplasmas were more likely to have fetal in- flammatory response syndrome and probably bronchopulmonaly dysplasia (4).

Two different biovars and 14 serovars of $\mathrm{Uu}$. have been isolated in humans. Biovar 1 includes serovars 1, 3, 6, 14 while biovars 2 includes serovars 2, 4, 5, and 7-13. Some studies have demonstrated that certain serovars or biovars 2 are more often associated with an adverse pregnancy outcome. Recently, Kim et al. demonstrated that the biovar diversity of Uu. in amniotic fluid was associated neither with a different pregnancy outcome nor with the magnitude of the intraamniotic inflammatory response (7). Mitsunary et al. showed that the difference between biovars in the cervix did not influence the evidence of preterm labor (10). A high density vaginal colonization with $\mathrm{Uu}$. was an independent risk factor for clinical chorioamnionitis and preterm delivery, while low density vaginal colonization levels had no effect (12). Also a high density vaginal Uu. colonization seemed to be an important factor in an ascending infection. Prophylactic erythromycin has not been shown to be of benefit, this finding may well be due to the limited transplacental transfer of macrolide antibiotics $(3,11)$.

\section{Conclusion}

We studied 225 women between 24 and 36 weeks of gestation with PPROM. In the control group the prevalence of colonization of the cervix with genital mycoplasmas during pregnancy was $32 \%$. In contrast, women with PPROM were found to have a cervical colonization rate with genital mycoplasmas of $96 \%$. Our results provide strong evidence of an association between cervical colonization with genital mycoplasmas and PPROM. Maternal serum CRP level did not correlate with histologically confirmed inflammatory changes in the placenta and membranes. We recommended early antibiotic treatment against the genital mycoplasmas in all the cases of PPROM.

\section{References}

1. Abele-Horn M, Schotz M, Wolf C, Kolben M. High density vaginal Ureaplasma urealyticum colonization as a risk factor for chorioamniontis and preterm delivery. Acta Obstet. Gynecol Scand 2000;79:973-8.

2. Carey JC, Blackwelder WC, Nugent RP. Antepartum cultures for Ureaplasma urealyticum are not useful in predicting pregnancy outcome. Am J Obstet Gynecol 1991;164:728-33

3. Eschenbach DA, Nugent RP, Rao AV, et al. A randomized placebo-controlled trial of erythromycin for the treatment of Ureaplasma urealyticum to prevent premature delivery. The Vaginal Infections and Prematurity Study Group. Am J Obstet Gynecol 1991;164:734-40

4. Goldenberg RL, Anders WW, Goepfert AR, Faye-Petersen O, Cliver SP, Carlo WA. The Alabama Preterm Birth Study: Umbilical cord blood Ureaplasma urealyticum and Mycoplasma hominis cultures in very preterm newborn infants. Am J Obstet Gynecol 2008;198:43e.1-43e5.

5. Gray JD, Robinson HB, Magne J, Thombson RB jr. Adverse outcome in pregnancy following amniotic fluid isolation of Ureaplasma Urealyticum. Prenat Diagn 1992;12:111-117.

6. Horowitz S, Horowitz M, Mazor M, Porath A Glezerman M. Ureaplasma urealyticum cervical colonization as a marker for pregnancy complications. Int $\mathrm{J}$ Gynecol Obstet 1995;48:15-19.

7. Kim M, Kim G, Romero R, Shim SS, Kim EC, Yoon BH. Biovar density of Ureaplasma Urealyticum in amniotic fluid. Distribution, intrauterine inflammatory response and pregnancy outcomes. J Perinat Med 2003;31:146-52.
Kace
strán
stránka
Compar
of PPRC
findi
o nadpis
+ ku
tive prot 
8. Li YH, Brauner A, Jonsson B. Ureaplasma Urealyticum induced production of proinflamatory cytokines by macrophages. Pediatr Res 2000;48:919-24.

9. McDonald HM, O'Loughlin JA, Jolley P. Prenatal microbiological risk factor associated with preterm birth. Br J Obstet Gynecol 1992;99:190-5.

10. Mitsunari M, Yoshida S, Deura I, et al. Cervical Ureaplasma urealyticum colonization might be associated with increased incidence of preterm delivery in pregnant women without prophlogistic microorganisms on routine examination. J Obstet Gynecol Res 2001;31:16-22.

11. Ogasawa KK, Goodwin TM. The efficacy of prophylactic erytromycin in preventing vertical transmission of Ureaplasma urealyticum. Am J Perinatol 1997;14:770-5.
12. Shim SS, Romero R, Hong JS. Clinical significance of intraamniotic inflammation in patients with preterm premature rupture of membranes. Am J Obstet Gynecol 2004;191:1339-45.

13. Watts DH, Krohn MA, Hillier S. The association of amniotic fluid infection with gestation age and neonatal outcome among women in preterm labor. Obstet Gynecol 1992;79:351-7.

14. Witt A, Berger A, Gruber J. Increased intrauterine frequency of Ureaplasma urealyticum in women with preterm labor and preterm premature of the membranes and subsequent caesarean delivery. Am J Obstet Gynecol 2005;193: $1663-9$

Received: 24/06/2009.

Accepted in revised form: 25/09/2009.

\section{Corresponding author:}

Marian Kacerovský, MD, Department of Obstetrics and Gynaecology, University Hospital, Sokolská 581, 50005 Hradec Králové, Czech Republic; e-mail: kacermar@fnhk.cz 